Heterogeneity in PFC-amygdala connectivity in middle childhood, and concurrent interrelations with inhibitory control and anxiety symptomology.

Kelley E. Gunther, Daniel Petrie, Alaina L. Pearce, Bari A. Fuchs, Koraly Pérez-Edgar, Kathleen L. Keller, \& Charles Geier

Penn State University

This study was funded by the National Science Foundation Graduate Research Fellowship Grant No. DGE1255832 (to KEG), the National Institute on Drug Abuse T32 DA017629 (to DP), and the National Institutes of Health, National Institute of Diabetes and Digestive and Kidney Diseases R01 DK110060 (to KLK). The content is the sole responsibility of the authors and does not necessarily represent the official position of any funding agencies. 


\begin{abstract}
The prefrontal cortex (PFC) is a key brain area in considering adaptive regulatory behaviors. This includes regulatory projections to regions of the limbic system such as the amygdala, where the nature of functional connections may confer lower risk for anxiety disorders. The PFC is also associated with behaviors like executive functioning. Inhibitory control is a behavior encompassed by executive functioning, and is generally viewed favorably for adaptive socioemotional development. Yet, some research suggests that high levels of inhibitory control may actually be a risk factor for some maladaptive developmental outcomes, like anxiety disorders. In a sample of 51 children ranging from 7-9 years old, we examined resting state functional connectivity between regions of the PFC and the amygdala. We used Subgrouping Group Iterative Multiple Model Estimation (S-GIMME) to identify and characterize data-driven subgroups of individuals with similar networks of connectivity between these brain regions. Generated subgroups were collapsed into children characterized by the presence or absence of recovered connections between the PFC and amygdala. We then tested whether inhibitory control, as measured by a stop signal task, moderated the relation between these subgroups and child-reported anxiety symptoms. We found an inverse relation between stop-signal reaction times and reported count of anxiety symptoms when controlling for connectivity group, suggesting that greater inhibitory control was actually related to greater anxiety symptoms, but only when accounting for patterns of PFC-amygdala connectivity. These data suggest that there is a great deal of heterogeneity in the nature of functional connections between the PFC and amygdala during this stage of development. The findings also provide support for the notion of high levels of inhibitory control as a risk factor for anxiety, but traitlevel biopsychosocial factors may be important to consider in assessing the nature of risk.
\end{abstract}




\section{Heterogeneity in PFC-amygdala connectivity in middle childhood, and concurrent interrelations with inhibitory control and anxiety symptomology.}

The prefrontal cortex (PFC) is broadly associated with a wide spectrum of regulatory tasks, including executive functioning and emotion regulation (Miller \& Cohen, 2001). Conventionally, the ability to regulate one's behavior is viewed as a mechanism for adaptive development, often understood as a positive relation between levels of regulation and positive outcomes, such that "more is better". However, recent data suggest that it may be possible to have too much of a good thing, and increased levels of inhibitory control, a sub-domain of executive functioning, may actually exacerbate risk for anxiety disorders (Carlson \& Wang, 2007; Eggum-Wilkens et al., 2016; Henderson et al., 2015; Henderson \& Wilson, 2017; Thorell et al., 2004; White et al., 2011). While it is difficult to quantify the exact prevalence, it is estimated that up to $28.3 \%$ of individuals worldwide are affected by anxiety disorders (Baxter et al., 2013), with an even larger portion impacted by symptomology below clinical threshold. Indeed, the approximate lifetime presence of anxiety disorders broadly construed is between 15 and $20 \%$, and onset is frequently in childhood (Beesdo et al., 2009). Thus, it is important to better understand the etiology of anxiety disorders, as well as mechanisms of risk and resilience through development.

To better characterize the complex association between inhibitory control and risk for anxiety, it is critical to better understand the neurological processes that contribute to risk for anxiety. Functional connections spanning the PFC and the amygdala, a limbic structure associated with the detection of emotionally salient stimuli, are associated with adaptive socioemotional development, including fewer anxiety symptoms (Gee et al., 2013; Tottenham \& Gabard-Durnam, 2017). As such, we tested whether the interaction between inhibitory control 
performance and resting state connections between regions of the amygdala and the PFC related to anxiety symptomology in a sample of school-age children.

\section{Executive functioning}

Executive functioning (EF) allows an individual to flexibly respond to stimuli even in the face of a competing prepotent response, supporting goal attainment (Diamond, 2006). EF is closely tied to the functioning of the PFC (Zelazo et al., 2008). The PFC has a protracted developmental trajectory, not reaching maturity until well into adolescence (Casey et al., 2005). As such, adult-like proficiency in executive functioning has a delayed emergence relative to many other domains of development (Welsh et al., 1991).

For the purposes of this manuscript, we consider EF through the lens of a three-factor model, dividing the broad umbrella term into the dissociable components of set shifting, working memory/updating, and inhibitory control (Miyake et al., 2000). However, these subdomains of EF are not necessarily a unitary construct. High levels of performance in one domain (i.e., set shifting) need not confer high levels of performance in another (i.e., inhibitory control; Blackwell et al., 2014).

Positive developmental outcomes typically associated with higher levels of executive functioning include increased school readiness (Fitzpatrick et al., 2014) and more sophisticated theory of mind (Carlson \& Moses, 2001) relative to peers. Impaired executive functioning is thought to be a transdiagnostic risk factor for a wide variety of clinical diagnoses (Snyder et al., 2015; Zelazo, 2020), including Attention Deficit Hyperactivity Disorder (ADHD; e.g. Barkley, 1997), Obsessive Compulsive Disorder (OCD; e.g. Shin et al., 2013) and bipolar disorder (i.e. Bora et al., 2009). Increased levels of attention shifting may operate as a protective mechanism 
against internalizing problems (Eggum-Wilkens et al., 2016; Henderson et al., 2015; Henderson \& Wilson, 2017, Toren et al., 2000; White et al., 2011). Similar findings have been reported for working memory (Basten et al., 2012; Moran, 2016). However, research remains mixed on whether inhibitory control, specifically, acts in the same protective fashion.

Supporting the traditional hypothesis that "more is better," some work finds an inverse relation between inhibitory control and internalizing symptoms in both children (Kooijmans et al., 2000; Lengua, 2003; Wolfe \& Bell, 2014) and adults (Ansari \& Derakshan et al., 2011; Basten et al., 2011). However, other research has found that increased inhibitory control may act as a risk factor for higher levels of internalizing behaviors, particularly among children (Carlson \& Wang, 2007; Eggum-Wilkens et al., 2016). As such, while undercontrol may relate to maladaptive developmental outcomes (Ansari \& Derakshan et al., 2011; Basten et al., 2011; Kooijmans et al., 2000; Lengua, 2003; Wolfe \& Bell, 2014), overcontrol may also be similarly deleterious (Carlson \& Wang, 2007; Eggum-Wilkens et al., 2016; Henderson et al., 2015; Henderson \& Wilson, 2017). Thus, the relation between inhibitory control and internalizing symptoms may be an inverted-U shape rather than linear. That is, both lower and higher levels of inhibitory control may relate to greater internalizing symptoms, and the optimal range of inhibitory control may instead be found at more moderate levels (Gunther \& Pérez-Edgar, 2021; Northoff \& Tumati, 2019).

A proposed mechanism underlying the possible inverted-U shaped association between inhibitory control and internalizing symptoms is that high levels of inhibitory control contribute to behavioral rigidity and overcontrol. Overcontrolled tendencies and the inability to effectively toggle between attentional states may potentiate maladaptive social behaviors, which may in turn 
promote negative affect, limit environmental engagement and learning, and ultimately increase risk for psychopathology (Gunther \& Pérez-Edgar, 2021; Henderson et al., 2015; Henderson \& Wilson, 2017). More work is needed to characterize and understand these relations and underlying mechanisms.

\section{The PFC, functional connections to the amygdala, and emotion regulation}

As EF is closely tied to the functioning of PFC (Zelazo et al., 2008) and amygdala is often considered a "salience detector," responding to stimuli important for adaptive engagement with one's environment (e.g., affective stimuli; Adolphs, 2008), neural circuitry between the amygdala and the PFC is critical for adaptive socioemotional processes (Ochsner et al., 2012). Functional connections between the amygdala and the PFC evolve over the course of development and continue through young adulthood (Taber-Thomas \& Pérez-Edgar, 2015). Indeed, the PFC develops on an extremely protracted timeline, not reaching maturity until young adulthood (Casey et al., 2005). Meanwhile, the amygdala reaches near-maturity in early childhood (Gabard-Durnam et al., 2018; Saygin et al., 2015). This temporal order of development suggests that while amygdala function is shaped early in life by environmental influences, the activity of the amygdala both influences the development of the PFC and shapes the nature of functional connections between the amygdala and PFC (Tottenham \& GabardDurnam, 2017). Animal work suggests that connections from the amygdala to the PFC emerge prior to the reciprocal connections, demonstrating this directional cascade in development (Bouwmeester et al., 2002a; Bouwmeester et al., 2002b; Tottenham \& Gabard-Durnam, 2017). Thus, the PFC itself as well as its circuitry to the amygdala are in flux through development and are integral to consider in assessing adaptive socioemotional development. 
Resting state connectivity analyses aim to assess functional connections in the brain, in the absence of a task (Uddin et al., 2010). Resting state data provide unique information regarding brain networks and circuits, highlighting spontaneous activity in these circuits rather than activity elicited by a task (Lv et al., 2018; Uddin et al., 2010). However, resting state measures have been relatively under-utilized in this area of research. Reflecting the available literature, here we review findings pertaining to functional connections between the amygdala and PFC that are task-elicited rather than examining networks at rest, demonstrating mixed findings on the relation between connectivity and adaptive behavior, and/or predominantly in adult samples. Thus, work is needed to more thoroughly characterize patterns of resting state connectivity that may confer risk for anxiety in childhood.

Gee and colleagues (2013) found in a healthy cross-sectional sample of children that the functional connectivity between the amygdala and PFC in response to fearful faces flips in directionality from positive to negative around 10 years of age. Corresponding with this finding, there were also age-related decreases in amygdala activity in response to fearful faces through childhood. These findings suggest that changes in fronto-amygdala connectivity may relate to the downregulation of the amygdala in response to fearful stimuli by dampening amygdala reactivity (Gee et al., 2013). Additionally, the directionality of the connection between the amygdala and the medial prefrontal cortex (mPFC) significantly mediated the relation between age and reported separation anxiety within the sample. Furthermore, when covarying for age, children with negative connectivity were more likely to have lower reported anxiety (Gee et al., 2013). Therefore, these age-related changes in task-based fronto-amygdala connectivity and negative fronto-amygdala connectivity may be critical in adaptive socioemotional development. 
As mentioned, findings are mixed when identifying patterns of resting state connectivity that characterize anxious individuals. Some work finds no significant differences in resting state connectivity between regions of the PFC and the amygdala as a function of anxiety (Prater et al., 2013). However, Hahn and colleagues (2011) found that clinically anxious adults may have decreased resting state connectivity between the left orbitofrontal cortex and the left amygdala compared to healthy controls, suggesting that less robust functional connections between these regions may be related to greater anxious symptomatology. Similarly, Liu and colleagues (2015) found that adolescents with a generalized anxiety disorder diagnosis showed decreased connectivity between the left amygdala and left dorsolateral prefrontal cortex (dlPFC) at rest.

On the other hand, Geiger and colleagues (2016) reported that socially anxious adults showed increased connectivity between the left orbitofrontal gyrus and the left amygdala. They also found dissociations of connectivity within the left executive control network, including increased connectivity within the left middle frontal gyrus and decreased connectivity within the left orbitofrontal gyrus. The authors suggest that the high levels of connectivity found in frontoamygdala circuits paired with low levels of connectivity in the executive control network may be capturing an imbalance between top-down and bottom-up control as part of the etiology of anxious symptomatology.

\section{Assessing resting state connectivity using GIMME}

Traditional network connectivity approaches (e.g., correlation-based), while valuable, are not able to determine directionality between brain regions of interest (ROIs; Smith et al., 2011). Group Iterative Multiple Model Estimation (GIMME), in contrast, is a statistical approach that reliably calculates direction of influence between ROIs on both an individual and group level (Gates \& Molenaar, 2012; Gates et al., 2014; Smith et al., 2011). The ability to consider the 
directionality of connections will help to elucidate any potential upregulatory or downregulatory connections from PFC to amygdala. Additionally, while traditional resting-state analytic approaches rely on the assumption of group homogeneity, GIMME accommodates heterogeneity in connectivity across individuals in the sample (Gates \& Molenaar, 2012; Gates et al., 2014). Eliminating the assumption of homogeneity may be more appropriate in examining functional connectivity during a resting state scan, which is less constrained.

In addition to being able to better model heterogeneity and directionality, GIMME also has subgrouping functionality (“S-GIMME”), which generates subgroups of individuals based on similarities in individual-level functional connectivity maps (Gates et al., 2017). This data-driven method allows for the identification and characterization of neural heterogeneity into categorical subgroups independent of behavioral phenotypes. For example, by applying S-GIMME to resting state data from a sample of children with and without ADHD, Gates and colleagues (2014) identified 5 subgroups of children with unique connectivity patterns amongst fronto-parietal ROIs. Two of the subgroups were composed overwhelmingly of children with an ADHD diagnosis. This suggests both heterogeneity amongst children with ADHD, but also a distinctiveness in connectivity patterns of children with ADHD versus typically developing children. These findings also suggest that using a data-driven grouping approach may provide a more nuanced way of characterizing populations above and beyond a clinical diagnosis (Gates et al., 2014).

Similarly, McCormick and colleagues (2019) collected MRI data during the Balloon Analogue Risk Task (BART; Lejuez et al., 2002) to assess risky decision-making in a sample of both children and adults. They found that the subgroups generated by S-GIMME using cortical 
and subcortical ROIs were different from the a priori adolescent, young adult, and adult groups. Moreover, data-derived and a priori groups were differentiated by distinct patterns of connections between ROIs during the task (McCormick et al., 2019). These papers suggest that S-GIMME may provide additional nuance in understanding profiles of risk above and beyond categorical variables conventionally used to describe a sample (Gates et al., 2014; McCormick et al., 2019). Thus, this grouping feature of GIMME will help us identify subgroups based on neural patterns, regardless of overt behavior, to test as a potential risk or protective factor for anxiety.

\section{Present study}

In the present study we interrogated if inhibitory control proficiency moderated the association between resting state PFC-amygdala connectivity and anxiety symptoms in 7-9-yearold children. Based on the reviewed literature, this stage of childhood may be just prior to a developmental shift from positive to negative connectivity between PFC and amygdala (Gee et al., 2013), suggesting it is an important time point in which to examine individual differences in connectivity as they pertain to symptoms of anxiety. Therefore, we first characterized patterns of resting state PFC-amygdala connectivity in a sample of children 7-8 years of age using SGIMME, an emerging method of generating data-driven subgroups of connectivity patterns (Gates et al., 2017). Due to the relative novelty of this method, we first sought to describe these subgroups and examine how these connectivity patterns related to both inhibitory control performance and child-reported anxiety symptoms. Then, we tested whether inhibitory control proficiency, a potential risk factor for anxiety, moderated the association between PFC-amygdala connectivity and anxiety symptoms in middle childhood. 
Although current work has produced inconsistent findings on the relation between functional connectivity and symptoms of anxiety (Geiger et al., 2016; Hahn et al., 2011; Liu et al., 2015; Prater et al., 2013), we predicted that children with no significant connections recovered between regions of the PFC and the amygdala will report greater levels of anxiety. Conversely, we posit that children with more dense patterns of recovered connections between the PFC and the amygdala will report lower levels of anxiety. We also predicted that children with significant functional connections between regions of the PFC and the amygdala would display better performance on our measure of inhibitory control, the stop signal task.

Finally, we predicted that that inhibitory control performance will positively moderate the relation between connectivity group and reported anxiety symptoms, where higher levels of inhibitory control will strengthen the relation between weak/negative PFC-amygdala connectivity and greater anxiety symptoms. In other words, children with resting state patterns not reflecting recovered connections between the amygdala and PFC, which may confer lower levels of regulation, will be more likely to show higher levels of reported anxiety if they also show higher levels of inhibitory control.

\section{Methods}

\section{Participants}

At time of data analysis, 77 children were enrolled in an ongoing longitudinal, familyrisk study originally designed to examine the neurocognitive determinants of pediatric obesity. The study consisted of six separate laboratory visits. Data included in this study were acquired across two different visits detailed below. 
Based on the family risk design used in the ongoing study, all children were healthy weight at enrollment (BMI-for-age $<85^{\text {th }} \%$ ), but were either "high risk" for obesity if both biological parents had BMIs in the overweight (i.e., father $>25 \mathrm{~kg} / \mathrm{m}^{2}$ ) or obese (i.e., mother $>$ $\left.30 \mathrm{~kg} / \mathrm{m}^{2}\right)$ range or "low risk" if both biological parents had BMIs in the healthy weight range $(<$ $25 \mathrm{~kg} / \mathrm{m}^{2}$ ). Participants were excluded if they had any neurodevelopmental disorders, colorblindness, claustrophobia, contraindications for functional magnetic resonance imaging (fMRI), did not speak English as a first language, if they were on any medications/had any medical diagnoses that may impact the child's appetite or food preferences/choices, were not of healthy body weight (body mass index for age $\%<85$ ), or if their parents did not meet the BMI criteria for high or low risk.

Of the enrolled participants, 64 completed a resting state fMRI scan. However, 11 children's scans could not be used due to errors in file writing that prevented the preprocessing pipeline to run to completion, and one participant was excluded for a diagnosis of ADHD after initial enrollment in the ongoing study. Therefore, the final sample included 51 children ranging from 7-9 years of age at the time of the fMRI $(\mathrm{M}=8.13, \mathrm{SD}=0.60,96.1 \%$ White, $58.8 \%$ female), but children were between 7-8 years old at time of recruitment. The median family income was between $\$ 76,000$ and $\$ 100,000.76 .5 \%$ of parents who filled out questionnaires had a Bachelor's degree or higher, and $68.6 \%$ of their partners had a Bachelor's degree or higher. Full details of the sample are described in Table 1. These demographics reflect the surrounding rural college town community. All study procedures were approved by the Institutional Review Board at the Pennsylvania State University. All parents and children completed written consent/assent and were compensated for their time. 
Table 1.

Table describing demographics of the final sample

\begin{tabular}{|c|c|c|c|}
\hline & $N$ & $\%$ & \\
\hline Sex (female) & 30 & 58.8 & \\
\hline Familial obesity risk status (high risk) & 19 & 37.3 & \\
\hline \multicolumn{4}{|l|}{ Race } \\
\hline White & 49 & 96.1 & \\
\hline Asian & 2 & 3.9 & \\
\hline \multicolumn{4}{|l|}{ Ethnicity } \\
\hline Not Hispanic or Latino & 52 & 100 & \\
\hline \multicolumn{4}{|l|}{ Yearly income } \\
\hline$\$ 20,000-\$ 35,999$ & 2 & 3.9 & \\
\hline$\$ 36,000-\$ 50,999$ & 5 & 9.8 & \\
\hline$\$ 51,000-\$ 75,999$ & 12 & 23.5 & \\
\hline$\$ 76,000-\$ 100,000$ & 11 & 21.6 & \\
\hline Over $\$ 100,000$ & 19 & 37.3 & \\
\hline Declined to respond & 2 & 3.9 & \\
\hline \multicolumn{4}{|l|}{ Primary parent education } \\
\hline High School or GED & 7 & 13.7 & \\
\hline Associate's Degree & 4 & 7.8 & \\
\hline Bachelor's Degree & 21 & 40.2 & \\
\hline Master's Degree & 11 & 21.6 & \\
\hline Ph.D. & 8 & 15.7 & \\
\hline \multicolumn{4}{|l|}{ Parent's partner's education } \\
\hline High School or GED & 8 & 15.7 & \\
\hline Associate's Degree & 1 & 2.0 & \\
\hline Tech or Vocational School & 5 & 9.8 & \\
\hline Bachelor's Degree & 18 & 35.3 & \\
\hline Master's Degree & 10 & 19.6 & \\
\hline J.D. & 1 & 1.9 & \\
\hline M.D. & 1 & 1.9 & \\
\hline Ph.D. & 5 & 9.8 & \\
\hline \multirow[t]{2}{*}{ Other/did not respond } & 2 & 3.9 & \\
\hline & Mean & SD & Range \\
\hline Age at scan & 8.13 years & 0.60 & $7.14-9.26$ \\
\hline
\end{tabular}

\section{Protocol}

Data included in this study were collected at visits two and six. State anxiety measurements were collected on visit two following a laboratory meal, while fMRI data and stop 
signal task were collected on visit six. For visit six, children were instructed to fast three hours prior to arrival, and once they arrived, rated their fullness using a child-appropriate visual analog scale (VAS; Keller et al., 2006). If children were $<25 \%$ full according to the VAS, they were given a small snack (apple juice and granola bar) so that they were in a neutral appetitive state during the fMRI. Before and after the MRI, children rated their state anxiety using the Children's Anxiety Meter - State (CAMS-S; Ersig et al., 2013). Following the fMRI, children completed a delay of gratification task, were offered a small snack (grapes, apple juice, cheese crackers), and rated images they saw in the scanner prior to completing the stop signal task.

In preparation for the MRI, children completed a two-session mock scanner protocol at visits four and five. As part of this protocol, children were able to explore the mock scanner, enter the bore, play a game on the computer screen, and complete movement training games. This training protocol has resulted in high success rates (i.e., $>90 \%$ usable functional scans) in prior studies with this age-group (Adise et al., 2018; English et al., 2018).

\section{Data acquisition}

Imaging data were acquired using a 3-Tesla MRI scanner (MAGNETOM Trio with Tim system, Siemens Medical Solutions, Erlangen, Germany). High resolution structural images were collected using a T1-weighted magnetization-prepared rapid acquisition gradient echo (MPRAGE) sequence to acquire 160 slices (1x1x1mm voxels). During this scan, children watched a video of baby animals to improve scan quality and reduce motion.

Immediately following the anatomical scan, resting state data were collected. During the resting state scan, children watched an abstract video (Vanderwal et al., 2015). Resting state functional images were collected using a T2*-weighted gradient single-shot blood-oxygen-level- 
dependent (BOLD) echo planar imaging (EPI) sequence to acquire 33 slices $(3 \times 3 \times 3 m m$ voxels, $\mathrm{TR}=2000 \mathrm{~ms}, \mathrm{TE}=26.0 \mathrm{~ms}$, flip angle $=90, \mathrm{FoV}=220 \times 220$, slice gap $=0 \mathrm{~mm})$. Both the anatomical scan and the resting state were the first two scans of a larger protocol that involved assessing children's responses to food images and office supplies. These data will be published in a forthcoming submission.

\section{Data preprocessing}

fMRIprep. Results included in this manuscript come from preprocessing performed using fMRIPrep 20.2.1 (Esteban, Markiewicz, et al., 2018; Esteban, Blair, et al., 2018; RRID:SCR_016216), which is based on Nipype 1.5.1 (Gorgolewski et al., 2011; Gorgolewski et al., 2018; RRID:SCR_002502).

T1-weighted (T1w) images were corrected for intensity non-uniformity (INU) with N4BiasFieldCorrection (Tustison et al., 2010), distributed with ANTs 2.3.3 (Avants et al., 2008, RRID:SCR_004757), and used as T1w-reference throughout the workflow. The T1w-reference was then skull-stripped with a Nipype implementation of the antsBrainExtraction.sh workflow (from ANTs), using OASIS30ANTs as target template. Brain tissue segmentation of cerebrospinal fluid (CSF), white-matter (WM) and gray-matter (GM) was performed on the brain-extracted T1w using fast (FSL 5.0.9, RRID:SCR_002823, Zhang, Brady, and Smith 2001). Volume-based spatial normalization to one standard space (MNI152NLin2009cAsym) was performed through nonlinear registration with antsRegistration (ANTs 2.3.3), using brainextracted versions of both $\mathrm{T} 1 \mathrm{w}$ reference and the $\mathrm{T} 1 \mathrm{w}$ template. The following template was selected for spatial normalization: ICBM 152 Nonlinear Asymmetrical template version 2009c [Fonov et al. (2009), RRID:SCR_008796; TemplateFlow ID: MNI152NLin2009cAsym], 
For each of the BOLD runs collected during resting state, the following preprocessing was performed. First, a reference volume and its skull-stripped version were generated using a custom methodology of fMRIPrep. A deformation field to correct for susceptibility distortions was estimated based on fMRIPreplu2019s fieldmap-less approach. The deformation field is that resulting from co-registering the BOLD reference to the same-subject $\mathrm{T} 1 \mathrm{w}$-reference with its intensity inverted (Wang et al., 2017; Huntenburg, 2014). Registration is performed with antsRegistration (ANTs 2.3.3), and the process regularized by constraining deformation to be nonzero only along the phase-encoding direction and modulated with an average fieldmap template (Treiber et al., 2016). Based on the estimated susceptibility distortion, a corrected EPI (echo-planar imaging) reference was calculated for a more accurate co-registration with the anatomical reference. The BOLD reference was then co-registered to the $\mathrm{T} 1 \mathrm{w}$ reference using flirt (FSL 5.0.9, Jenkinson \& Smith, 2001) with the boundary-based registration (Greve \& Fischl, 2009) cost-function.

Co-registration was configured with nine degrees of freedom to account for distortions remaining in the BOLD reference. Head-motion parameters with respect to the BOLD reference (transformation matrices, and six corresponding rotation and translation parameters) are estimated before any spatiotemporal filtering using mcflirt (FSL 5.0.9, Jenkinson et al., 2002). BOLD runs were slice-time corrected using 3dTshift from AFNI 20160207 (Cox \& Hyde, 1997, RRID:SCR_005927). The BOLD time-series (including slice-timing correction when applied) were resampled onto their original, native space by applying a single, composite transform to correct for head-motion and susceptibility distortions. These resampled BOLD time-series will be referred to as preprocessed BOLD in original space, or just preprocessed BOLD. The BOLD 
time-series were resampled into standard space, generating a preprocessed BOLD run in MNI152NLin2009cAsym space.

First, a reference volume and its skull-stripped version were generated using a custom methodology of fMRIPrep. Several confounding time-series were calculated based on the preprocessed BOLD: framewise displacement (FD), DVARS and three region-wise global signals. FD was computed using two formulations following Power (absolute sum of relative motions, Power et al. (2014)) and Jenkinson (relative root mean square displacement between affines, Jenkinson et al. 2002)). FD and DVARS are calculated for each functional run, both using their implementations in Nipype (following the definitions by Power et al. 2014). The three global signals are extracted within the CSF, the WM, and the whole-brain masks.

Additionally, a set of physiological regressors were extracted to allow for componentbased noise correction (CompCor, Behzadi et al. 2007). Principal components are estimated after high-pass filtering the preprocessed BOLD time-series (using a discrete cosine filter with 128s cut-off) for the two CompCor variants: temporal (tCompCor) and anatomical (aCompCor). tCompCor components are then calculated from the top $2 \%$ variable voxels within the brain mask. For aCompCor, three probabilistic masks (CSF, WM and combined CSF+WM) are generated in anatomical space. The implementation differs from that of Behzadi et al. in that instead of eroding the masks by 2 pixels on BOLD space, the aCompCor masks are subtracted a mask of pixels that likely contain a volume fraction of GM. This mask is obtained by thresholding the corresponding partial volume map at 0.05 , and it ensures components are not extracted from voxels containing a minimal fraction of GM. 
Finally, these masks are resampled into BOLD space and binarized by thresholding at 0.99 (as in the original implementation). Components are also calculated separately within the WM and CSF masks. For each CompCor decomposition, the k components with the largest singular values are retained, such that the retained componentslu2019 time series are sufficient to explain 50 percent of variance across the nuisance mask (CSF, WM, combined, or temporal). The remaining components are dropped from consideration.

The head-motion estimates calculated in the correction step were also placed within the corresponding confounds file. The confound time series derived from head motion estimates and global signals were expanded with the inclusion of temporal derivatives and quadratic terms for each (Satterthwaite et al. 2013). Frames that exceeded a threshold of $0.5 \mathrm{~mm}$ FD or 1.5 standardized DVARS were annotated as motion outliers. All resamplings can be performed with a single interpolation step by composing all the pertinent transformations (i.e., head-motion transform matrices, susceptibility distortion correction when available, and co-registrations to anatomical and output spaces). Gridded (volumetric) resamplings were performed using antsApplyTransforms (ANTs), configured with Lanczos interpolation to minimize the smoothing effects of other kernels (Lanczos 1964). Non-gridded (surface) resamplings were performed using mri_vol2surf (FreeSurfer).

Many internal operations of fMRIPrep use Nilearn 0.6.2 (Abraham et al. 2014, RRID:SCR_001362), mostly within the functional processing workflow. More details of the pipeline can be seen the section corresponding to workflows in fMRIPrep $\backslash u 2019$ s documentation. 
XCP Engine. XCP Engine cleans and parses time series data from the preprocessed fMRIprep data. In this processing, we selected the 36P+DESPIKE to clean for motion artifacts (Lydon-Staley et al., 2018). From XCP Engine, we derived time series data for each of our ROIs. For our analysis we a priori selected bilateral ROIs encompassed by the prefrontal cortex, which was central to our hypothesis: the frontal pole, frontal medial cortex, frontal orbital cortex, and the amygdala. ROIs were anatomically defined by the Harvard Oxford atlas, used in prior studies of similar age range and brain areas of interest (i.e., Liu et al., 2021).

\section{Resting state connectivity}

GIMME was used to construct functional connectivity maps during resting state fMRI (Gates \& Molenaar, 2012; Gates et al., 2014; Smith et al., 2011). GIMME operates within a unified structural equation model framework (uSEM; Gates, Molenaar, Hillary, Ram, \& Rovine, 20120; Kim, Zhu, Chang, Bentler, \& Ernst, 2007), where a data-driven algorithm constructs functional connectivity maps by using information from both the individual- and group-level through a process of model building and model pruning. First, information is pooled across all participants and used to create a connectivity map that is representative of the sample using modification indices (Sorbom, 1989). Group paths are retained if they improve model fit, according to modification indices, at a user-defined cutoff value. Following recommendations from Gates and colleagues (2021), we lowered the default group level threshold from the default $75 \%$ to $51 \%$. This reflects criteria specifying that greater than half of individuals must have a path present and improve overall model fit for the path to be represented in the group level map.

Importantly, all autoregressive paths are freely estimated in order to determine directionality between ROI paths by establishing Granger causality (Granger, 1969; Lane \& Gates, 2017). The group map is then pruned by eliminating paths which, because of adding paths 
across iterations, no longer improve model fit. Next, individual-level paths are estimated. For each individual, the group-level model is fit, and then modification indices are used to assess whether additional paths for each individual would improve the fit of the model. This iterative procedure continues until excellent model fit is obtained. Excellent model fit is defined as exceeding two out of four fit indices (Brown, 2006; Gates \& Molenaar 2012): root mean squared of approximation $(\mathrm{RMSEA}) \leq 0.05$; standardized root mean residual $(\mathrm{SRMR}) \leq 0.05$; comparative fit index $(\mathrm{CFI}) \geq 0.95$; non-normed fit index $(\mathrm{NNFI}) \geq 0.95$.

We allowed GIMME to freely construct subgroups based on individual connectivity maps. With this approach, the researcher does not need to specify the number of subgroups. Rather, the best fit for number of subgroups is chosen by the algorithm (Gates et al., 2017; McCormick et al., 2019). S-GIMME decides the number of subgroups by creating a similarity matrix using the individual estimates of the group-level connections (Gates et al., 2017). Then, the similarity matrix is subjected to the Walktrap community detection approach (Pons \& Latapy, 2006), which arrives at subgroups by utilizing data-driven random walks to maximize modularity. Thus, subgroups are based on the characteristics of the individual-level connectivity maps. Walktrap approaches have been shown to avoid spurious subgroupings and do not rely on significance testing which is a common problem in network contexts (Gates et al., 2016). After S-GIMME identifies distinct subgroups, unique subgroup specific paths are identified in a similar way as the group- and individual-level paths. We retained the default $50 \%$ as the subgroup level cutoff, reflecting criteria specifying that half of the individuals within a designated subgroup must have a path present for the path to be represented on the subgroup level map. In sum, the final solution from S-GIMME includes group-, subgroup-, and individuallevel paths that are all estimated at the individual-level. 


\section{Inhibitory control}

Inhibitory control was assessed on visit 6 , following the MRI scan. Children were offered a small snack (i.e., cheese crackers, grapes and apple juice) between the MRI and SST so that they were in a neutral appetitive state during the task. The stop signal task assesses inhibitory control by measuring the latency of response inhibition. The current version of the stop signal task used was developed specifically for this study to assess individual differences in children's inhibitory control in response to food images that varied by portion size (i.e., large versus small) and energy density (i.e., high calorie versus low calorie). However, in the current analyses, we focused only on children's overall performance (i.e., independent of the food condition they were responding to). The stop signal task was administered using Matlab2018b and Psychtoolbox3 (Kleiner et al., 2007). Pictures of plates of food were presented with a triangle folded napkin on either the left or right side of the plate (go-signal; see Figure 1) for $1500 \mathrm{~ms}$ with an interstimulus-interval of $50 \mathrm{~ms}$ (i.e., fixation). Children were asked to sort the plates according to which side of the plate the napkin was on and to press the left or right arrow keys when the napkin appeared on the left or right side of the plate, respectively. They were encouraged to respond as quickly as possible. Children were also told that the plate would be sometimes get covered with a silver cloche (i.e., stop-signal; see Figure 1) and were instructed not to respond if the silver cloche appeared. The silver cloche (i.e., stop-signal) was presented after variable delay on ( $25 \%$ of trials). The variable stop-signal delay (SSD) was determined by a step-wise adaptive procedure which increased the SSD 50ms after each successful stop trial and reduced the SSD by $50 \mathrm{~ms}$ after each unsuccessful stop trial with the first $\mathrm{SSD}=250 \mathrm{~ms}$. This adaptive procedure maintained $\sim 0.50$ probability of successful response inhibition. The task parameters were based 
on specifications articulated by Verbruggen and colleagues (2019) for best practices in measuring stop-signal reaction time according to the theoretical racehorse model.

The task consisted of 256 trials across of 4 randomized blocks (i.e., 64 trials per block).. After the practice, children were reminded to respond quickly and told that they may be encouraged to respond quickly during the task. To prevent children from slowing throughout the task, the message 'Faster' was presented after trials in which they responded slower than 1.5 standard deviations below their mean reaction time in practice. Between each block, children were given the opportunity to take a break and were shown their average response time (e.g., 'How Fast Were You?'), given encouragement for how quickly they were responding (e.g., 'Wow, that is faster than a second'), and were reminded not to press the arrow keys if the silver cloche covered the plate.

Stop-signal reaction times (SSRT) were computed using the integration method after replacing omitted go responses with the participants' slowest go RT (Verbruggen et al., 2019). SSRT was only computed if the assumptions of the racehorse model were met (i.e., mean go RT $>$ mean unsuccessful stop RT; Verbruggen et al., 2019). Of the 51 children who provided resting state data, 44 children provided SSRT data. Additionally, 1 participant's SSRT was excluded for not meeting the assumptions of the race model, and was therefore also treated as missing data. 

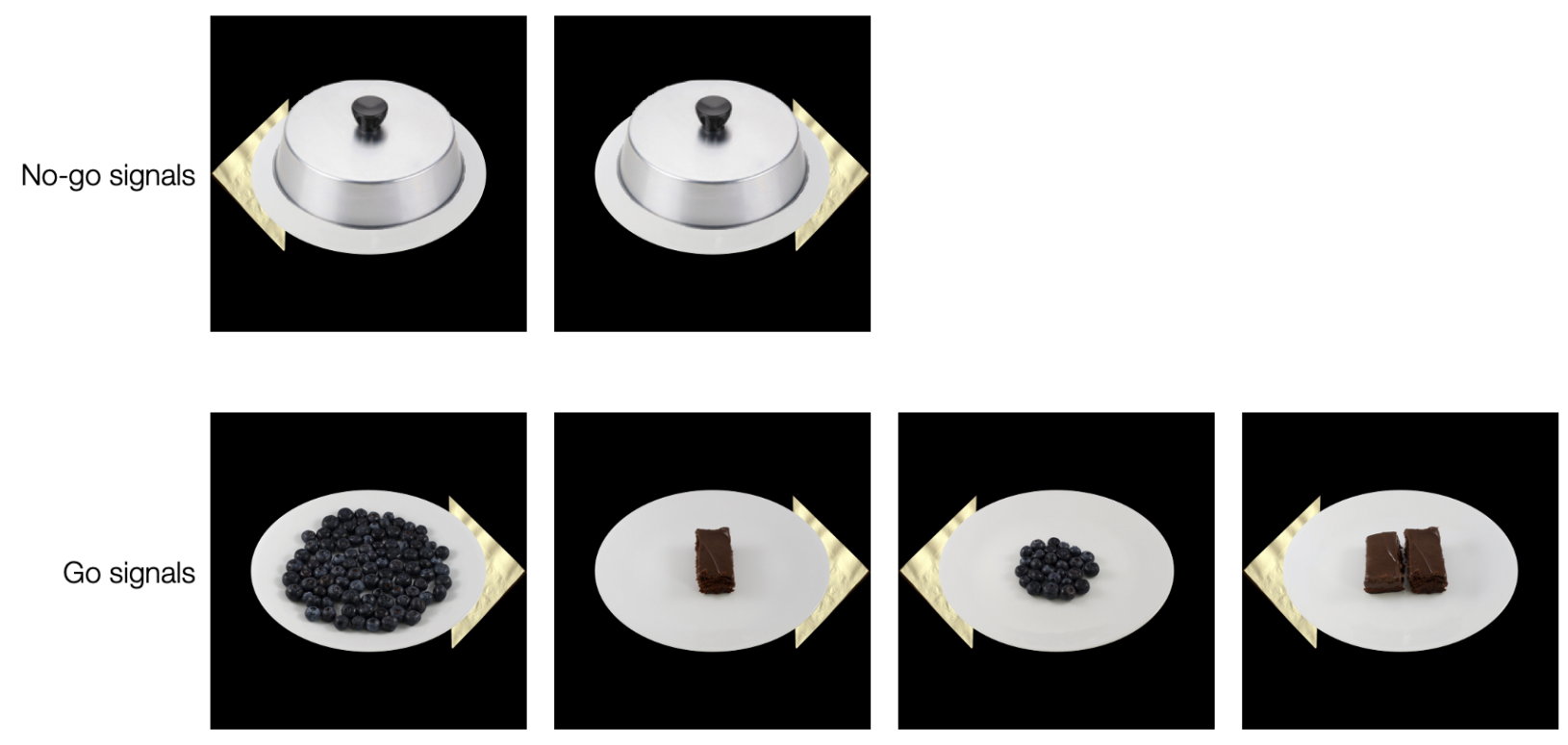

Figure 1. Schematic depicting stop signal task.

\section{Anxiety symptoms}

Participants reported on their own anxiety symptoms using the Revised Children's

Manifest Anxiety Scale (RCMAS), a 37-item questionnaire that asks children to endorse whether they do or do not experience a behavior (example: "I have trouble making up my mind").

Children filled out this questionnaire at their second laboratory visit. The RCMAS correlates highly with the trait anxiety subscale of the State-Trait Anxiety Inventory for children (STAIC), but not the state subscale, demonstrating construct validity with trait anxiety (Reynolds, 1980). The RCMAS is a reasonably stable measure of anxiety in school-age populations (Reynolds, 1981), and demonstrates good test-retest reliability (Wisniewski et al., 1987). We were interested in trait anxiety in contrast to state anxiety; it is the stable, persistent symptomology that is of 
greater clinical relevance than ordinary intraindividual fluctuations in anxiety (Beauchaine, 2015).

For our analysis we used the total anxiety score of the RCMAS, which ranged from 0 to 28. Endorsing 19 or more behaviors $(\mathrm{N}=6)$ indicates clinically significant levels of anxiety (Stallard et al., 2001). Forty five of the 51 children providing resting state data provided data from the RCMAS. Within our sample, this score had excellent internal consistency (Cronbach's Alpha $=0.89)$.

Additionally, we collected information on the child's level of state anxiety immediately before the MRI using the Children's Anxiety Meter - State (CAMS-S; Ersig et al., 2013). For this measure, children are presented with a thermometer-like graphic and are asked to mark where they feel right now, with the bottom annotated as "Calm, not nervous or worried" and the top annotated as "Very very nervous or worried." The child's mark is then translated into a numeric value, with 0 representing lowest levels of state anxiety and 10 representing highest levels of state anxiety. Fifty of the 51 children providing resting state data also provided CAMS data.

\section{Data analysis}

All statistical analyses were conducted in R version 3.6.2. S-GIMME was used to generate data-driven subgroups characterizing resting state connectivity between the amygdala and regions of the PFC. In all analyses, familial obesity risk status ("high risk" if maternal BMI $>30 \mathrm{~kg} / \mathrm{m}^{2}$ and paternal $\mathrm{BMI}>25 \mathrm{~kg} / \mathrm{m}^{2}$ ), age, and sex were all treated as covariates. We also entered pre-scan state anxiety as a covariate in these models to control for the child's mental state upon entering the scanner (Ersig et al., 2013). 
Using group memberships derived from S-GIMME, we tested group differences in anxiety, as measured by the RCMAS, and in SSRT, as a metric of inhibitory control. Finally, we tested whether SSRT moderated the association between connectivity group membership and anxiety symptoms.

\section{Results}

S-GIMME identified five subgroups in the sample. The number of members in each subgroups can be found in Table 2.

Table 2.

Table displaying number of children in each S-GIMME generated subgroup.

\begin{tabular}{ccc}
\hline Subgroup & $N$ & Final group \\
\hline 1 & 13 & 1 \\
2 & 15 & 2 \\
3 & 7 & 1 \\
4 & 6 & 2 \\
5 & 10 & 2 \\
\hline
\end{tabular}

To assess model fit for the final solution, we used recommendations published by Gates and Molenaar (2012). The average root mean squared error of approximation (RMSEA) was 0.10 , the average standardized root mean-square residual (SRMR) was 0.04 , the average nonnormed fit index (NNFI) was 0.92, and the and the average comparative fit index (CFI) was 0.96. Because the SRMR $<.05$ and CFI $>.95$, we deemed the model fit excellent (Gates \& Molenaar, 2012; Hu \& Bentler, 1999). 
Our hypotheses were central to functional connections between the amygdala and regions of the PFC, and we had no hypotheses specific to intra-region PFC connectivity or connections between the amygdala with more specific regions of the PFC. Therefore, we collapsed across subgroups that $\operatorname{did}(\mathrm{N}=2)$ and $\operatorname{did} \operatorname{not}(\mathrm{N}=3)$ recover significant connections between the amygdala and regions of the PFC for analysis (Figure 2). Thus, group membership was included in models as a dichotomous variable. For these final groups, group 1 (characterized by the presence of recovered connections between the amygdala and regions of the PFC) included 20 individuals, and group 2 (characterized by the absence of recovered connections between the amygdala and PFC) included 31 individuals. There were no significant differences in age, sex, or familial risk for obesity between these groups. 

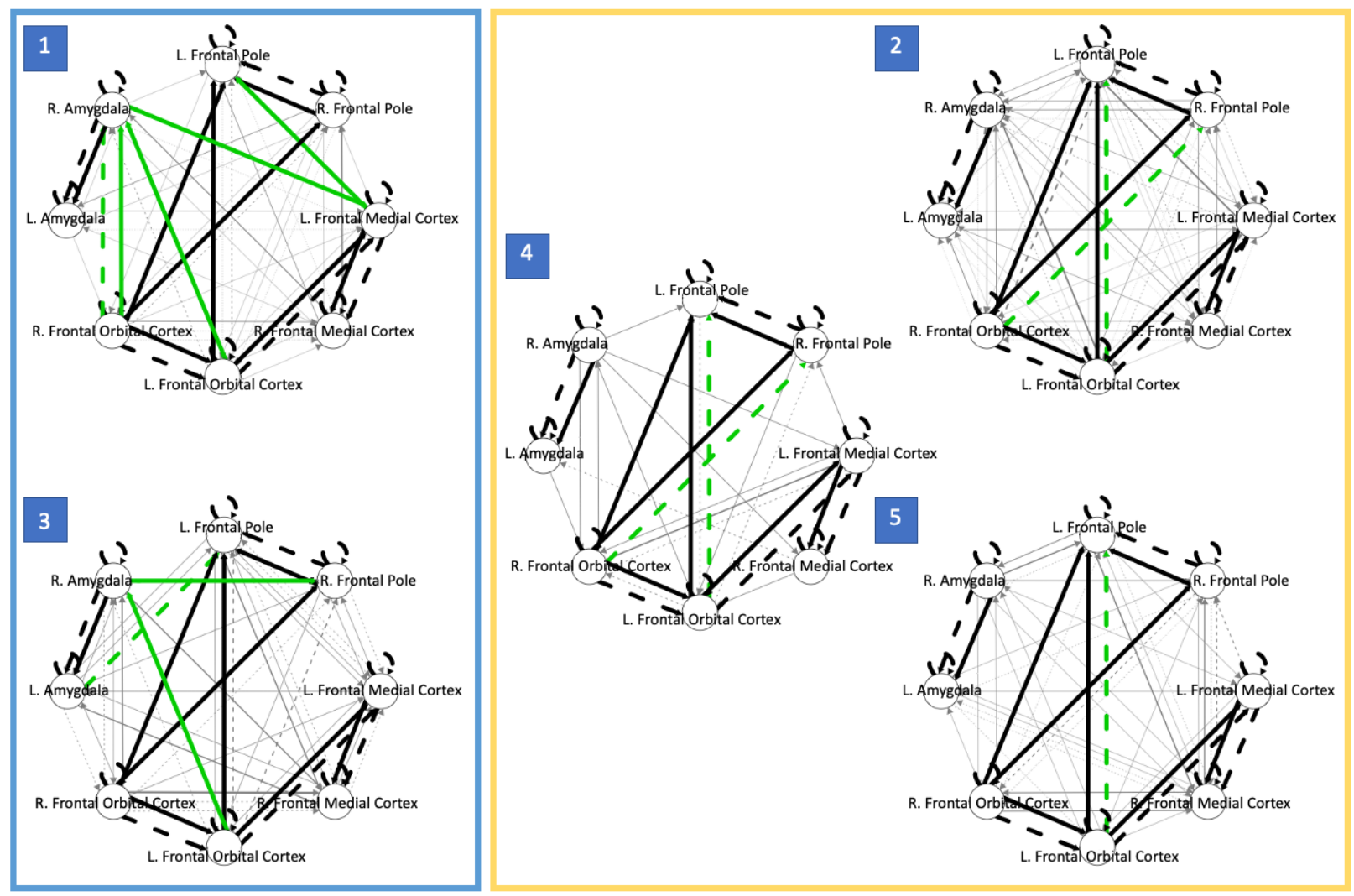

Figure 2. Subgroups recovered by S-GIMME $(\mathrm{N}=5)$. Grey lines represent individual-level connections, black lines represent connections characteristic of the entire sample, and green lines represent connections that define the subgroup. Solid lines represent contemporaneous effects and dashed lines represent lagged effects. Subgroups were collapsed to create two groups: 1) characterized by the recovery of significant connections between the amygdala and regions of the PFC (blue box) and 2) characterized by the absence of recovery of significant connections between the amygdala and regions of the PFC (yellow box).

First, we assessed differences in anxiety and SSRT in the groups derived by S-GIMME. Count of anxiety symptoms and their distribution, faceted by connectivity group, are plotted in figure 3 . We statistically tested whether connectivity group membership was related to anxiety symptoms, while controlling for sex, age, pre-scan state anxiety, and obesity risk. In assessing 
relations to anxiety symptoms, we used a negative binomial distribution to account for the countnature of the variable, as well as overdispersion within the sample (Cameron \& Trivedi, 1990). There were no significant relations between connectivity group and number of reported anxiety symptoms. Results from the model can be seen in Table 3.

Next, we tested whether connectivity group membership was related to SSRT, while controlling for sex, age, pre-scan state anxiety, and obesity risk. SSRT data and distribution, faceted by connectivity group, are plotted in Figure 3. A normal distribution was used in this analysis. There was a significant negative association between connectivity group membership and SSRT, $b(S E)=-55.54(24.84), p=.03$. That is, individuals in characterized by no recovered connections between the amygdala and regions of the PFC (group 2), had faster SSRTs than individuals characterized by the presence of recovered connections between the amygdala and regions of the PFC (group 1). This pattern suggests better inhibitory control for members of group 2. Results from the model can be seen in Table 3. 

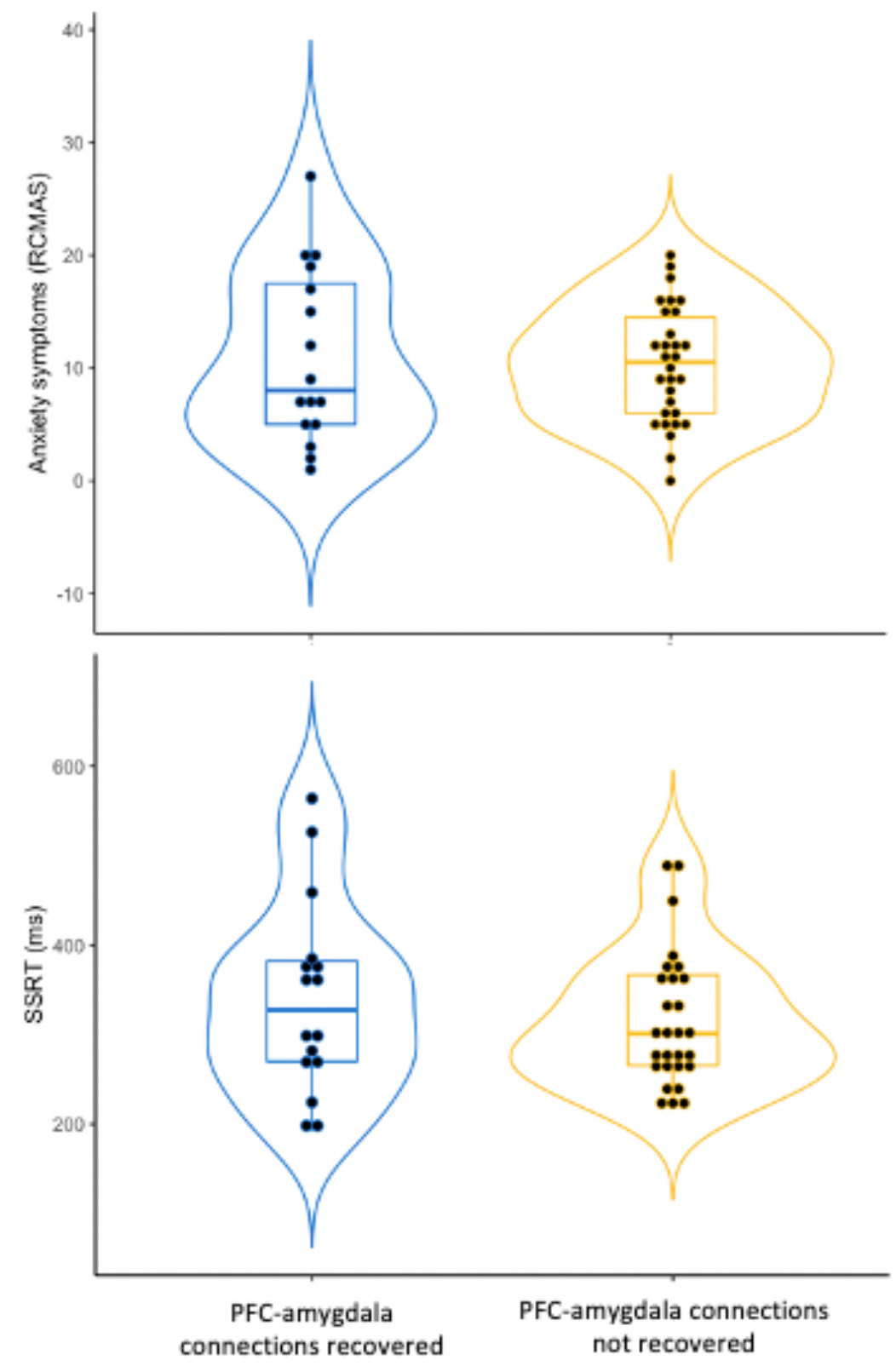

Figure 3. Violin plot depicting the mean, range, and distribution of both SSRT and self-reported anxiety symptoms, split by connectivity group. 
Table 3.

Negative binomial and linear models assessing relations between connectivity group and selfreported anxiety symptoms via the RCMAS and inhibitory control performance indexed by SSRT, respectively. Odds included for model ran using a negative binomial distribution. Sex, age, prescan state anxiety, and obesity risk category were entered as covariates in all models.

\begin{tabular}{|c|c|c|c|c|c|c|c|}
\hline \multirow[b]{2}{*}{ Parameter } & \multirow[b]{2}{*}{ Estimate } & \multicolumn{3}{|c|}{$\begin{array}{c}\text { Anxiety symptoms } \\
\text { (RCMAS) }\end{array}$} & \multicolumn{3}{|c|}{ SSRT } \\
\hline & & $S E$ & Odds ratio & z-value & Estimate & $S E$ & t-value \\
\hline Intercept & 1.44 & 1.40 & 4.22 & 1.02 & $1026.52 * * *$ & 166.10 & 6.18 \\
\hline Sex & -0.15 & 0.20 & 0.86 & -0.77 & -5.72 & 23.92 & -0.24 \\
\hline Age & 0.09 & 0.17 & 1.09 & 0.57 & $-82.69 * * *$ & 19.59 & -4.22 \\
\hline $\begin{array}{l}\text { Familial obesity } \\
\text { risk status }\end{array}$ & 0.23 & 0.19 & 1.26 & 1.20 & $58.42 *$ & 24.73 & 2.36 \\
\hline $\begin{array}{l}\text { Pre-scan state } \\
\text { anxiety }\end{array}$ & 0.07 & 0.04 & 1.07 & 1.56 & -7.12 & 4.97 & -1.43 \\
\hline $\begin{array}{l}\text { Connectivity } \\
\text { group }\end{array}$ & -0.07 & 0.20 & 0.93 & -0.32 & $-55.54^{*}$ & 24.84 & -2.24 \\
\hline
\end{tabular}

$+p<.10, * p<.05, * * p<.01, * * * p<.001$

Sex: $0=$ female, $1=$ male

Familial obesity risk status: $0=$ low risk, $1=$ high risk

Connectivity group: 1 = Amygdala-PFC connections recovered, 2 = Amygdala-PFC connections not recovered

We finally tested whether SSRT moderated the relation between connectivity group and anxiety symptoms, also using a negative binomial distribution. We found no significant interaction between SSRT and connectivity group, $b(S E)=0.003(0.002), p>.05$. However, we did find a significant conditional effect of SSRT on anxiety symptoms $b(S E)=-0.005(0.002), p$ $=.007)$. A follow-up analysis found that while controlling for group membership, there was a significant effect of SSRT on self-reported anxiety symptoms, $b=-0.002, p=.048$ (Table 5). That is, for each for each 1ms increase in SSRT (indicating less proficient inhibitory control), the expected log count of the number of reported anxiety symptoms decreases by 0.003 . The relation between SSRT and anxiety symptoms faceted by connectivity group is visualized in Figure 4. 
Table 4.

Negative binomial model assessing the interaction between SSRT and connectivity group on selfreported anxiety symptoms. Sex, age, pre-scan state anxiety, and obesity risk category were entered as covariates.

\begin{tabular}{lllll}
\hline Parameter & Estimate & SE & Odds ratio & z-value \\
\hline Intercept & $5.89^{* *}$ & 2.15 & 361.41 & 2.73 \\
Sex & -0.20 & 0.20 & 0.82 & -1.02 \\
Age & -0.25 & 0.21 & 0.78 & -1.20 \\
Familial obesity risk status & 0.31 & 0.18 & 1.36 & 1.50 \\
Pre-scan state anxiety & 0.02 & 0.04 & 1.02 & 0.44 \\
Connectivity group & $-1.24+$ & 0.72 & 0.29 & -1.73 \\
SSRT & $-0.005^{* *}$ & 0.002 & 0.995 & -2.67 \\
Connectivity group x SSRT & 0.003 & 0.002 & 1.003 & 1.58 \\
\hline
\end{tabular}

$+p<.10, * p<.05, * * p<.01, * * * p<.001$

Sex: $0=$ female, $1=$ male

Familial obesity risk status: $0=$ low risk, $1=$ high risk

Connectivity group: 1 = Amygdala-PFC connections recovered, 2 = Amygdala-PFC connections not recovered

Table 5.

Negative binomial model assessing the effect of SSRT on self-reported anxiety symptoms. Odds ratio included in parentheses. Sex, age, pre-scan state anxiety, obesity risk category, and connectivity group were entered as covariates.

\begin{tabular}{lllll}
\hline Parameter & Estimate & SE & Odds ratio & z-value \\
\hline Intercept & $4.70^{*}$ & 2.10 & 109.95 & 2.26 \\
Sex & -0.19 & 0.20 & 0.83 & -0.94 \\
Age & -0.20 & 0.21 & 0.82 & -0.93 \\
Familial obesity risk & 0.34 & 0.21 & 1.40 & 1.59 \\
status & & & & \\
Pre-scan state anxiety & 0.04 & 0.04 & 1.04 & 0.81 \\
Connectivity group & -0.16 & 0.23 & 0.85 & -0.71 \\
SSRT & $-0.003^{*}$ & 0.001 & 0.997 & -1.97 \\
\hline
\end{tabular}

$+p<.10, * p<.05, * * p<.01, * * * p<.001$

Sex: $0=$ female, $1=$ male

Familial obesity risk status: $0=$ low risk, $1=$ high risk

Connectivity group: 1 = Amygdala-PFC connections recovered, 2 = Amygdala-PFC connections not recovered 


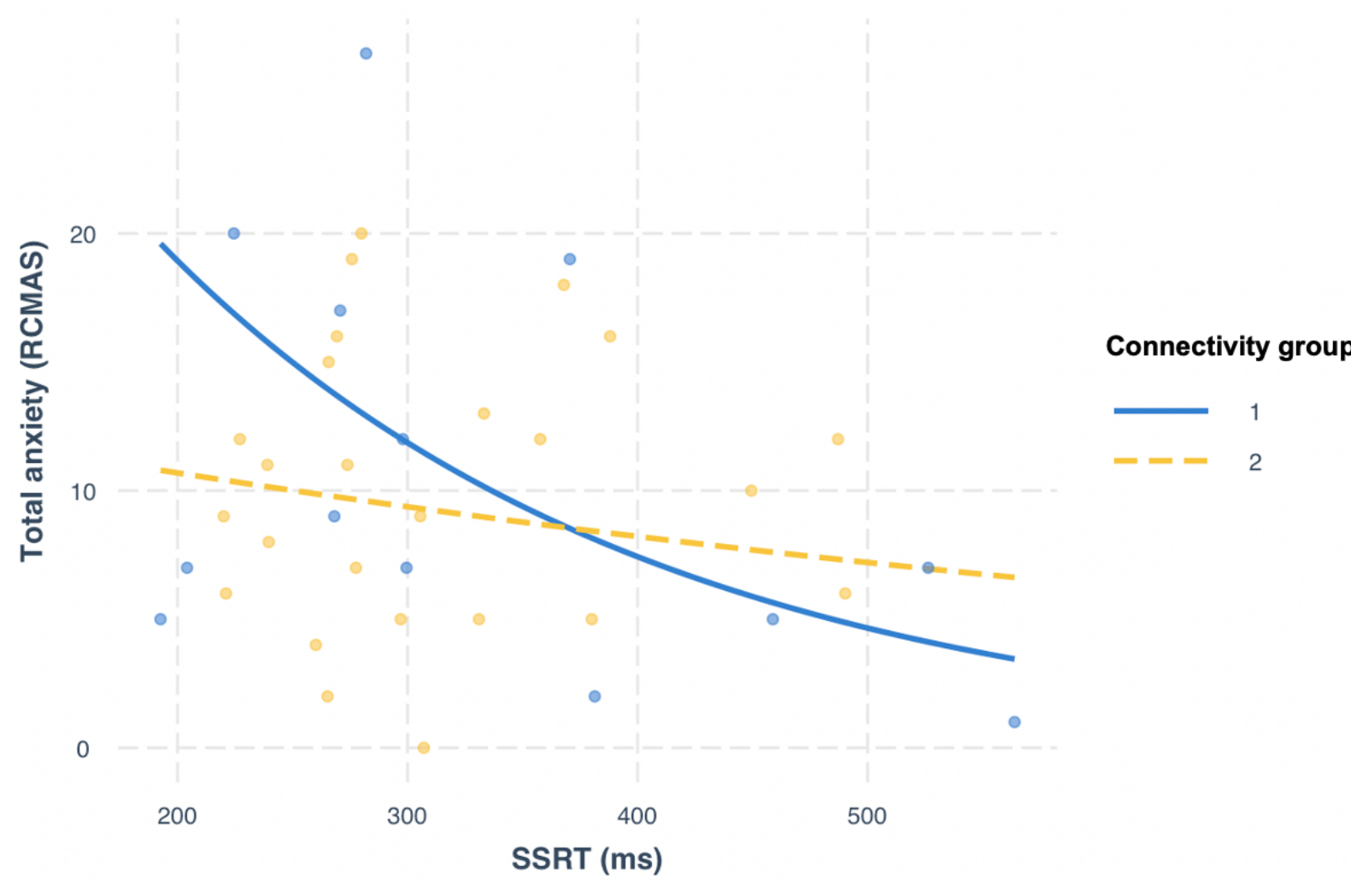

Figure 4. Plot depicting negative effect of SSRT on self-reported anxiety symptoms, faceted by connectivity group membership.

\section{Discussion}

In this study, we tested the interaction between inhibitory control performance and patterns of resting state PFC-amygdala connectivity in relation to anxiety symptoms. To do so, we used a data-driven approach to identify subgroups of children with unique patterns of PFCamygdala resting state connectivity.

S-GIMME freely constructs subgroups without a priori specification of number of desired subgroups. Thus, the finding of five subgroups within this sample highlights the heterogeneity of patterns of PFC-amygdala connectivity in this developmental window, 
especially in a sample unselected for anxiety or anxiety risk. We also note that the majority of children within this sample (31 of 51) were assigned to the subgroups where significant connections between the amygdala and regions of the PFC were not recovered. Middle childhood is a period of significant change for both the PFC and its functional connections (Casey et al., 2005; Gee et al., 2013; Tottenham \& Gabard-Durnam, 2017). The heterogeneity noted here may be capturing a period of large developmental change, or it may be highlighting heterogeneity in these circuits that persists through the lifespan. Future research should address this question empirically by conducting similar analyses on resting state data in either a cross sectional or longitudinal sample, focusing on the years surrounding age 10 where this purported shift in directionality of connectivity between the amygdala and PFC takes place (Gee et al., 2013).

We collapsed across these five subgroups to yield two groups for analysis that were more central to our research question. Group one was characterized by individuals in the subgroups where S-GIMME recovered connections between the amygdala and regions of the PFC, while group two was characterized by individuals without amygdala-PFC connections. These groups did not differ on age, sex, or familial obesity risk.

Additionally, we tested whether these groups differed on their reported anxiety symptoms or inhibitory control performance. Children characterized by no recovered connections between the amygdala and regions of the PFC had more proficient inhibitory control than children who did show these connections. However, we found no significant association between connectivity group membership and child-reported anxiety symptoms. Of note, we used self-reported anxiety symptoms rather than parent-report, and future research may be strengthened by incorporating both child and parent report of anxiety symptoms. While future research should attempt replication in a larger sample to see if this relation is robust, this association suggests that 
children for whom S-GIMME recovered connections between the amygdala and regions of the PFC may have less proficient inhibitory control, reflected in longer SSRTs. It may be the case that these recovered connections between the amygdala and PFC reflect rigidity in cognitive processing, and an inability to flexibly respond to task demands. Future work should address this association more directly, and in a larger sample, to examine this potential mechanistic link between PFC-amygdala resting state connectivity and inhibitory control.

Finally, integrating SSRT, anxiety, and connectivity group membership into the same model, we found that greater SSRTs, reflecting less proficient inhibitory control, were related to fewer self-reported anxiety symptoms, but only when controlling for connectivity group. This finding is consistent previous work suggesting that high levels of control may not always be adaptive for healthy development. Additionally, the fact that the interrelation between SSRT and anxiety symptoms was evident only when accounting for patterns of resting state brain connectivity is in line with the idea that trait-level biopsychosocial factors, like temperament, may further explain the links between executive functioning and socioemotional processes (Henderson et al., 2015; Henderson \& Wilson, 2017; White et al., 2011)

We recognize that this sample was not nationally representative, as children were majority white and high SES, as well as from highly educated families. Additionally, this sample was predominantly composed of children who were not clinically anxious. Only 6 of the 52 children reported clinically-relevant symptom levels. This may be due in part to the fact that while anxiety disorders may be diagnosed as young as preschool-age (Franz et al., 2013), onset of clinical-level symptomology may also be as late as adolescence or adulthood depending on the specific diagnosis (Beesdo et al., 2009). A slightly older sample or following children longitudinally may also offer more variability in anxiety symptomology. 
We should interpret these findings based on a number of methodological considerations, including age-related differences in cognitive state while an individual is instructed to rest during a resting state scan. For example, for a younger child, resting state scans may elicit greater levels of cognitive control as they suppress a desire to move or talk during the scan. Network properties may reflect this heightened state of cognitive control. Inconsistencies in prior work may in part reflect the unstructured nature of a resting state scan, and findings should consider the fact that the actual "task" of laying still in a scanner may vary systematically across development (Camacho et al., 2020). Furthermore, we recognize that parcellation is an important methodological decision, especially in assessing resting state connectivity (Bryce et al., 2021), and these results are specific to anatomic brain regions defined by the Harvard-Oxford atlas. Our decision to use the Harvard-Oxford atlas was informed by prior work with similar research questions and samples (e.g., Liu et al., 2021), however different parcellations have the potential to yield different results than reported in this manuscript.

Additionally, while prior theoretical and empirical work suggests that the relation between inhibitory control and symptoms of anxiety may be nonlinear, we chose to model a linear relation. Quadratic modeling between variables relies on the assumption that individuals were not sampled along a normal distribution, with oversampling from individuals at the extremes of a bell curve. Because our sampling scheme did not consider this, we were not equipped to reliably model quadratic relations between variables. Future work should intentionally sample from low and high levels of both anxiety and inhibitory control to accurately assess quadratic relations between these constructs (Northoff \& Tumati, 2019).

In conclusion, this study provides support of the notion that high levels of inhibitory control may relate to higher levels of self-reported anxiety symptoms as early as middle 
childhood. However, within this sample, these associations were evident when controlling for patterns of functional connections between the amygdala and PFC. Thus, it may be important to consider neural circuitry and behavior in characterizing risk for anxiety within middle childhood. 


\section{References}

Alexandre, A., Pedregosa, F., Eickenberg, M., Gervais, P., Mueller, A., Kossaifi, J., Gramfort, A., Thirion, B., \& Varoquaux, G. (2014). Machine Learning for Neuroimaging with Scikit-Learn. Frontiers in Neuroinformatics, 8. https://doi.org/10.3389/fninf.2014.00014.

Avants, B.B., Epstein, C. L., Grossman, M., \& Gee, J. C. (2008). Symmetric Diffeomorphic Image Registration with Cross-Correlation: Evaluating Automated Labeling of Elderly and Neurodegenerative Brain. Medical Image Analysis 12(1), 26-41. https://doi.org/10.1016/j.media.2007.06.004.

Adise, S., Geier, C. F., Roberts, N. J., White, C. N., \& Keller, K. L. (2018). Food or money? Children's brains respond differently to rewards regardless of weight status. Pediatric Obesity, 14(2), e12469. https://doi.org/10.1111/ijpo.12469

Adolphs, R. (2008). Fear, faces, and the human amygdala. Current Opinion in Neurobiology, 18, 166-172. https://doi.org/10.1016/j.conb.2008.06.006

Ansari, T. L., \& Derakshan, N. (2011). The neural correlates of impaired inhibitory control in anxiety. Neuropsychologia, 49, 1146-1153. https://doi.org/10.1016/j.neuropsychologia.2011.01.019

Basten, U., Stelzel, C., \& Fiebach, C. J. (2012). Trait anxiety and the neural efficiency of manipulation in working memory. Cognitive, Affective, and Behavioral Neuroscience, 12, 571-588. https://doi.org/10.3758/s13415-012-0100-3

Baxter, A. J., Scott, K. M., Vos, T., \& Whiteford, H. A. (2013). Global prevalence of anxiety disorders: a systematic review and meta-regression. Psychological medicine, 43(5), 897910 
Beauchaine, T. P. (2015) Future Directions in Emotion Dysregulation and Youth Psychopathology. Journal of Clinical Child \& Adolescent Psychology, 45(5), 875-896. https://doi.org/10.1080/15374416.2015.1038827

Beesdo, K., Knappe, S., \& Pine, D. S. (2009). Anxiety and Anxiety Disorders in Children and Adolescents: Developmental Issues and Implications for DSM-V. The Psychiatric clinics of North America, 32(3), 483-524. https://doi.org/10.1016/j.psc.2009.06.002

Behzadi, Y., Restom, K., Liau,, J., \& Liu, T. T. (2007). A Component Based Noise Correction Method (CompCor) for BOLD and Perfusion Based fMRI. NeuroImage 37(1), 90-101. https://doi.org/10.1016/j.neuroimage.2007.04.042.

Bouwmeester, H., Smits, K., \& Van Ree, J. M. (2002). Neonatal development of projections to the basolateral amygdala from prefrontal and thalamic structures in rat. Journal of Comparative Neurology, 450(3), 241-255. https://doi.org/10.1002/cne.10321

Bouwmeester, H., Wolterink, G., \& Van Ree, J. M. (2002). Neonatal development of projections from the basolateral amygdala to prefrontal, striatal, and thalamic structures in the rat. Journal of Comparative Neurology, 442(3), 239-249. https://doi.org/10.1002/cne.10084

Bryce, N. V., Flournoy, J. C., Guassi Moreira, J. F., Rosen, M. L., Sambook, K. A., Mair, P., \& McLaughlin, K. A. (2021). Brain parcellation selection: An overlooked decision point with meaningful effects on individual differences in resting-state functional connectivity. NeuroImage, 243, 118487. https://doi.org/10.1016/j.neuroimage.2021.118487. 
Camacho, M. C., Quiñones-Camacho, L. E., \& Perlman, S. B. (2020). Does the child brain rest?: An examination and interpretation of resting cognition in developmental cognitive neuroscience. Neuroimage, 212, 116688. https://doi.org/10.1016/j.neuroimage.2020.116688

Cameron, A. C., \& Trivedi, P. K. (1990). Regression-based tests for overdispersion in the Poisson model. Journal of econometrics, 46(3), 347-364. https://doi.org/10.1016/03044076(90)90014-K

Carlson, S. M., \& Moses, L. J. (2001). Individual differences in inhibitory control and children's theory of mind. Child Development, 72(4), 1032-1053. https://doi.org/10.1111/14678624.00333

Carlson, S. M., \& Wang, T. A. (2007). Inhibitory control and emotion regulation in preschool children. Cognitive Development, 22, 489-510. https://doi.org/10.1016/j.cogdev.2007.08.002

Casey, B. J., Tottenham, N., Liston, C., \& Durston, S. (2005). Imaging the developing brain: What have we learned about cognitive development? TRENDS in Cognitive Sciences, 9(3), 104-110. https://doi.org/10.1016/j.tics.2005.01.011

Cisler, J. M., \& Olatunji, B. O. (2012). Emotion regulation and anxiety disorders. Current psychiatry reports, 14(3), 182-187. https://doi.org/10.1007/s11920-012-0262-2

Cox, R. W., \& Hyde, J. S. (1997). Software Tools for Analysis and Visualization of fMRI Data. NMR in Biomedicine 10(4-5), 171-178. https://doi.org/10.1002/(SICI)10991492(199706/08)10:4/5<171::AID-NBM453>3.0.CO;2-L. 
Diamond, A. (2006). The early development of executive functions. In E. Bialystok \& F. Craik (Eds.), Lifespan Cognition: Mechanisms of Change (pp. 70-95). New York: Oxford University Press.

Eggum-Wilkens, N. D., Reichenberg, R. E., Eisenberg, N., \& Spinard, T.L. (2016). Components of effortful control and their relations to children's shyness. International Journal of Behavioral Development, 40(6), 544-554. https://doi.org/10.1177/0165025415597792

English, L. K., Masterson, T. D., Fearnbach, S. N., Tanofsky-Kraff, M., Fisher, J., Wilson, S. J., Rolls, B. J., \& Kelley, K. L. (2019). Increased brain and behavioural susceptibility to portion size in children with loss of control eating. Pediatric Obesity, 14(2), https://doi.org/10.1111/ijpo.12436.

Ersig, A. L., Kleiber, C., McCarthy, A. M., \& Hanrahan, K. (2013). Validation of a clinically useful measure of children's state anxiety before medical procedures. Journal for Specialists in Pediatric Nursing, 18(4), 311-319. https://doi.org/10.1111/jspn.12042

Esteban, O., Markiewicz, C., Goncalves, M., DuPre, E., Kent, J. D., Salo, T., Ciric, R., Pinsard, B., Blair, R. W., Poldrack, R. A., \& Gorgolewski, K. J. (2018). FMRIPrep. Software. Zenodo. https://doi.org/10.5281/zenodo.852659

Esteban, O., Markiewicz, C., Blair, R. W., Moodie, C., Isik, A. I., Erramuzpe, A., Kent, J., Goncalves, M., DuPre, E., Snyder, M., Oya, H., Ghosh, S. S., Wright, J., Durnezm J., Poldrack, R. A., \& Gorgolewski, K. J. (2018). MRIPrep: A Robust Preprocessing Pipeline for Functional MRI. Nature Methods, 16, 111-116. https://doi.org/10.1038/s41592-018-0235-4. 
Fonov, VS, AC Evans, RC McKinstry, CR Almli, and DL Collins. 2009. Iu201cUnbiased Nonlinear Average Age-Appropriate Brain Templates from Birth to Adulthood.lu201d NeuroImage 47, Supplement 1: S102. https://doi.org/10.1016/S1053-8119(09)70884-5.

Franz, L., Angold, A., Copeland, W., Costello, E. J., Towe-Goodman, N., \& Egger, H. (2013) Preschool Anxiety Disorders in Pediatric Primary Care: Prevalence and Comorbidity. Journal of the American Academy of Child and Adolescent Psychiatry, 52(12), 12941303. http://doi.org/10.1016/j.jaac/2013.09.008

Gabard-Durnam, L. J., O’Muircheartaigh, J., Dirks, H., Dean III, D. C., Tottenham, N., \& Deoni, S. (2018). Human amygdala functional network development: A cross-sectional study from 3 months to 5 years of age. Developmental cognitive neuroscience, 34, 63-74. https://doi.org/10.1016/j.den.2018.06.004

Gates, K. M., Fisher, Z., \& Bollen, K. A. (2021). Latent Variable GIMME. Psychological Methods, 25(2), 227-242. https://doi.org/10.1037/met0000229

Gates, K. M., Lane, S. T., Varangis, E., Giovanello, K., \& Guiskewicz, K. (2017). Unsupervised Classification During Time-Series Model Building. Multivariate Behavioral Research, 52(2), 129-148. https://doi.org/10.1080/00273171.2016.1256187

Gates, K. M. \& Molenaar, P. C. M. (2012). Group search algorithm recovers effective connectivity maps for individuals in homogenous and heterogenous samples. Neuroimage, 63, 310-319. https://doi.org/10.1016/j.neuroimage.2012.06.026

Gates, K. M., Molenaar, P. C. M., Iyer, S. P., Nigg, J. T., \& Fairm D. A. (2014). Organizing Heterogeneous Samples Using Community Detection of GIMME-Derived Resting State Functional Networks. PLoS ONE, 9(3), e91322. https://doi.org/10.1371/journal.pone.0091322 
Gee, D. G., Humphreys, K. L., Flannery, J., Goff, B., Telzer, E. H., Shapiro, M., Hare, T. A., Bookheimer, S. Y., \& Tottenham, N. (2013). A Developmental Shift from Positive to Negative Connectivity in Human Amygdala-Prefrontal Circuitry. Journal of Neuroscience, 33(10), 4584-4593. https://doi.org/10.1523/JNEUROSCI.3446-12.2013

Geiger, M. J., Domschke, K., Ipser, J., Hattingh, C., Baldwin, D. S., Lochner, C., \& Stein, D. J., (2016). Altered executive control network resting-state connectivity in social anxiety disorder. The World Journal of Biological Psychiatry, 17(1), 47-57. https://doi.org/10.3109/15622975.2015.1083613

Gorgolewski, K., Burns, C. D., Madison, C., Clark, D., Halchenko, Y. O., Waskom, M. L., \& Ghosh. S. (2011). Nipype: A Flexible, Lightweight and Extensible Neuroimaging Data Processing Framework in Python. Frontiers in Neuroinformatics 5(13). https://doi.org/10.3389/fninf.2011.00013.

Granger, C. W. (1969). Investigating causal relations by econometric models and cross-spectral methods. Econometrica: journal of the Econometric Society, 424-438.

Greve, D. N \& Fischl, B. (2009). Accurate and Robust Brain Image Alignment Using BoundaryBased Registration. NeuroImage 48(1), 63-72. https://doi.org/10.1016/j.neuroimage.2009.06.060.

Gunther, K. E. \& Pérez-Edgar, K. (2021). Dopaminergic Associations between Behavioral Inhibition, Executive Functioning, and Anxiety in Development. Developmental Review, 60, 100966. https://doi.org/10.1016/j.dr.2021.100966

Hahn, A., Stein, P., Windischberger, C., Weissenbacher, A., Spindelegger, C., Moser, E., Kasper, S., \& Lanxenberger, R. (2011). Reduced resting-state functional connectivity 
between amygdala and orbitofrontal cortex in social anxiety disorder. Neuroimage, 56, 881-889. https://doi.org/j.neuroimage.2011.02.064

Henderson, H. A., Pine, D. S., \& Fox, N. A. (2015). Behavioral inhibition and developmental risk: A dual-processing perspective. Neuropsychopharmacology, 40, 207-224. https://doi.org/10.1038.npp.2014.189

Henderson, H. A., \& Wilson, M. J. G. (2017). Attention processes underlying risk and resilience in behaviorally inhibited children. Current Behavioral Neuroscience Reports, 4, 99-106. https://doi.org/10.1007/s40473-017-0111-z

Hu, L. T., \& Bentler, P. M. (1999). Cutoff criteria for fit indexes in covariance structure analysis: Conventional criteria versus new alternatives. Structural equation modeling: a multidisciplinary journal, 6(1), 1-55. http://doi.org/10.1080/10705519909540118

Huntenburg, J. M. (2014). Evaluating Nonlinear Coregistration of BOLD EPI and T1w Images. Master's Thesis, Berlin: Freie Universität. http://hdl.handle.net/11858/00-001M-0000002B-1CB5-A.

Jenkinson, M, Bannister, P., Brady, M., \& Smith, S. (2002). Improved Optimization for the Robust and Accurate Linear Registration and Motion Correction of Brain Images. NeuroImage, 17(2): 825-841. https://doi.org/10.1006/nimg.2002.1132.

Jenkinson, M \& Smith, S. (2001). A Global Optimisation Method for Robust Affine Registration of Brain Images. Medical Image Analysis 5(2): 143-156. https://doi.org/10.1016/S13618415(01)00036-6.

Keller, K. L., Assur, S. A., Torres, M., Lofink, H. E., Thornton, J.C., Faith, M.S., \& Kissileff, H.R. (2006). Potential of an analog scaling device for measuring fullness in children: 
development and preliminary testing. Appetite, 47(2), 233-243. https://doi.org/10.1016/j.appet.2006.04.004

Kleiner, M., Brainard, D., \& Pelli, D. (2007). What's new in Psychtoolbox-3?.

Kooijmans, R., Scheres, A., \& Oosterlaan, J. (2000). Response inhibition and measures of psychopathology: a dimensional analysis. Child Neuropsychology, 6(3), 145-184. https://doi.org/10.1076/chin.6.3.175.3154

Lanczos, C. (1964). Evaluation of Noisy Data. Journal of the Society for Industrial and Applied Mathematics Series B Numerical Analysis, 1(1), 76-85. https://doi.org/10.1137/0701007. Lane, S. T. \& Gates, K. M. (2017). Automated selection of robust individual-level structural equation models for time series data. Structural Equation Modeling: A Multidisciplinary Journal, 24(5), 768-782. https://doi.org/10.1080/10705511.2017.1309978

Lejuez, C. W., Read, J. P., Kahler, C. W., Richards, J. B., Ramsey, S. E., Stuart, G. L., ... \& Brown, R. A. (2002). Evaluation of a behavioral measure of risk taking: the Balloon Analogue Risk Task (BART). Journal of Experimental Psychology: Applied, 8(2), 75. https://doi.org/10.1037/1076-898X.8.2.75

Lengua, L. J. (2003). Associations among emotionality, self-regulation, adjustment problems, and positive adjustment in middle childhood. Applied Developmental Psychology, 24, 595-618. https://doi.org/10.1016/j.appdev.2003.08.002

Liu, P., Vandermeer, M. R. J., Ali, O. M., Daoust, A. R., Joanisse, M. F., Barch, D. M., \& Hayden, E. P. (2021). Maternal Depression, Child Temperament, and Early-Life Stress Predict Never-Depressed Preadolescents' Functional Connectivity During a NegativeMood Induction. Clinical Psychological Science.

https://doi.org/10.1177/21677026211016419 
Lv, H., Wang, Z., Tong, E., Williams, L. M., Zaharchuk, G., Zeineh, M., Goldstein-Piekarski, A. N., Ball, T. M., Liao, C., \& Wintermark, M. (2018). Resting-state functional MRI: everything that nonexperts have always wanted to know. American Journal of Neuroradiology, 39(8), 1390-1399. https://doi.org/10.3174/ajnr.A5527

McCormick, E, M., Gates, K, M., \& Telzer, E. H. (2019). Model-based network discovery of developmental and performance-related differences during risky decision-making. Neuroimage, 188, 456-464. https://doi.org/10.1016/j.neuroimage.2018.12.042

Miller, E. K. \& Cohen, J. D. (2001). An Integrative Theory of Prefrontal Cortex Function. Annual Review of Neuroscience, 24, 167-202.

Moran, T. P. (2016). Anxiety and working memory capacity: A meta-analysis and narrative review. Psychological Bulletin, 142(8), 1-34. https://doi.org/10.1037/bul0000051

Northoff, G., \& Tumati, S. (2019). “Average is good, extremes are bad" - Non-linear inverted Ushaped relationship between neural mechanisms and functionality of mental features. Neuroscience and Biobehavioral Reviews, 104, 11-25. https://doi.org/10.1016/j.neubiorev.2019.06.030

Ochsner, K. N., Silvers, J. A., \& Buhle, J. T. (2012). Functional imaging studies of emotion regulation: a synthetic review and evolving model of the cognitive control of emotion. Annals of the New York Academy of Sciences, 1251, E1-24. https://doi.org/10.1111/j.1749-6632.2012.06751.x

Pons, P. \& Latapy, M. (2006). Computing communities in large networks using random walks. Journal of Graph Algorithms and Applications, 10(2), 191-218. 
Power, J. D., Mitra A., Laumann, T. O., Snyder, A. Z., Schlaggar, B. L., , \& Petersen, S. E. (2014). Methods to Detect, Characterize, and Remove Motion Artifact in Resting State fMRI. NeuroImage, 84(Supplement C), 320-341. https://doi.org/10.1016/j.neuroimage.2013.08.048.

Reynolds, C. R. (1980). Concurrent validity of What I Think and Feel: The Revised Children's Manifest Anxiety Scale. Journal of Consulting and Clinical Psychology, 48(6), 774775. https://doi.org/10.1037/0022-006X.48.6.774

Reynolds, C. R. (1981). Long-term stability of scores on the Revised-Children's Manifest Anxiety Scale. Perceptual and Motor Skills, 53(3), 702. https://doi.org/10.2466/pms.1981.53.3.702

Satterthwaite, T. D., Elliott, M. A., Gerraty, R. T., Ruparel, K., loughead, J., Calkins, M. E., Eikhoff, S. B., Hakonarson, H., Gur, R. C., Gur, R. E., \& Wolf, D. H. (2013). An improved framework for confound regression and filtering for control of motion artifact in the preprocessing of resting-state functional connectivity data. Neuroimage, 64, 240256. https://doi.org/10.1016/j.neuroimage.2012.08.052

Saygin, Z. M., Osher, D. E., Koldewyn, K., Martin, R. E., Finn, A., Saxe, R., Gabrieli, J. D. E., \& Sheridan, M. (2015). Structural Connectivity of the Developing Human Amygdala. PLoS ONE, 10(4), e0125170. https://doi.org/10.1371/journal.pone.0125170

Smith, S. M., Miller, K. L., Salimi-Khorshidi, G., Webster, M., Beckmann, C. F., Nichols, T. E., Ramsey, J. D., Woolrich, M. W. (2011). Network modeling methods for FMRI. Neuroimage, 54(2), 875-891. https://doi.org/10.1016/j.neuroimage.2010.08.063 
Stallard, P., Velleman, R., Langsford, J., \& Baldwin, S. (2001). Coping and psychological distress in children involved in road traffic accidents. British Journal of Clinical Psychology, 40(2), 197-208.

Taber-Thomas, B., \& Pérez-Edgar, K. (2015). Brain development in emerging adulthood. In J. Arnett (Ed), Oxford Handbook of Emerging Adulthood, 126-141. Oxford University Press

Thorell, L. B., Bohlin, G., \& Rydell, A. (2004). Two types of inhibitory control: Predictive relations to social functioning. International Journal of Behavioral Development, 28(3), 193-203. https://doi.org/10.1080/01650250344000389

Toren, P., Sadeh, M., Wolmer, L., Eldar, S., Koren, S., Weizman, R., \& Laor, N. (2000). Neurocognitive correlates of anxiety disorders in children: A preliminary report. Journal of Anxiety Disorders, 14(3), 239-247. https://doi.org/10.1016/s0887-6185(99)00036-5

Tottenham, N. \& Gabard-Durnam, L. J. (2017). The developing amygdala: a student of the world and a teacher of the cortex. Current Opinion in Psychology, 17, 55-60. https://doi.org/10.1016/j.copsyc.2017.06.012

Treiber, J. M., White, N. S., Steed, T. C., Bartsch, H., Holland, D., Farid, D., McDonald, C. R., Carter, B. S., Dale, A. M., \& Chen, C. C. (2016). Characterization and Correction of Geometric Distortions in 814 Diffusion Weighted Images. PLOS ONE, 11(3): e0152472. https://doi.org/10.1371/journal.pone.0152472.

Tustison, N. J., Avants, B. B., Cook, P. A., Zheng, Y., Egan, A., Yushkevich, P. A. \& Gee, J. C. (2010). N4ITK: Improved N3 Bias Correction. IEEE Transactions on Medical Imaging, 29(6): 1310-1320. https://doi.org/10.1109/TMI.2010.2046908. 
Ulfig, N., Setzer, M., \& Bohl, J. (2003). Ontogeny of the human amygdala. Annals of the New York Academy of Sciences, 985(1), 22-33.

Uddin, L. Q., Supekar, K., \& Menon, V. (2010). Typical and atypical development of functional human brain networks: insights from resting-state FMRI. Frontiers in systems neuroscience, 4, 21. https://doi.org/10.3389/fnsys.2010.00021

Vanderwal, T., Kelly, C., Eilbott, J., Mayes, L. C., \& Castellanos, F. X. (2015). Inscapes: A movie paradigm to improve compliance in functional magnetic resonance imaging. NeuroImage, 122, 222-232. https://doi.org/10.1016/j.neuroimage.2015.07.069

Verbruggen, F., Aron, A. R., Band, G. P. H., Beste, C., Bissett, P. G., Brockett, A. T., Brown, J. W., Chamberlain, S. R., Chambers, C. D., Colonius, H., Colzato, L. S., Corneil, B. D., Coxon, J. P., Dupuis, A., Eagle, D. M., Garavan, H., Greenhouse, I., Heathcode, A., Huster, R. J., Jahfari, S., Kenemans, J. L., Leunissen, I., Li, C. R., Logan, G. D., Matzke, D., Morein-Zamir, S., Murthy, A., Paré, M., Poldrack, R. A., Ridderinkhof, K. R., Robbins, T. W., Roesch, M., Rubia, K., Schacher, R. J., Schall, J. D., Stock, A., Swann, N. C., Thakkar, K. N., van der Molen, M. W., Vermeylen. L., Vink, M., Wessel, J. R., Whelan, R., Zandbelt, B. B., \& Boehler, C. N. (2019). A consensus guide to capturing the ability to inhibit actions and impulsive behaviors in the stop-signal task. eLife, 8, e46323. https://doi.org/10.7554/eLife.46323

Wang, S., Peterson, D. J., Gatenby, J. C., Li, W., Grabowski, T. J., \& Madhyastha, T. M. (2017). Evaluation of Field Map and Nonlinear Registration Methods for Correction of Susceptibility Artifacts in Diffusion MRI. Frontiers in Neuroinformatics, 11. https://doi.org/10.3389/fninf.2017.00017. 
Weintraub, S., Dikmen, S. S., Heaton, R. K., Tulsky, D. S., Zelazo, P. D., Bauer, P. J., Carlozzi, N. E., Slotkin, J., Blitz, D., Wallner-Allen, K., Fox, N. A., Beaumont, J. L., Mungas, D., Nowinski, C. J., Richler, J., Deocampo, J. A., Anderson, J. E., Manly, J. J., Borosh, B., Havlik, R., Conway, K., Edwards, E., Freund, L., King, J. W., Moy, C., Eitt, E., \& Gershon, R. C. (2013). Cognition assessment using the NIH Toolbox. Neurology, 80(11 Suppl 3), S54-S64. https://doi.org/10.1212/WNL.0b013e3182872ded

Welsh, M. C., Pennington, B. F., \& Groisser, D. B. (1991) A normative-developmental study of executive function: A window on prefrontal function in children. Developmental Neuropsychology, 7(2), 131-149. https://doi.org/10.1080/87565649109540483

White, L. K., McDermott, J. M., Degnan, K. A., Henderson, H. A., \& Fox, N. A. (2011). Behavioral Inhibition and anxiety: The moderating roles of inhibitory control and attention shifting. Journal of Abnormal Child Psychology, 39, 735-747. https://doi.org/10.1007/s10802-011-9490-x

Wisniewski, J. J., Genshaft, J. L., Mulick, J. A., \& Coury, D. L. (1987). Test-retest reliability of the revised children's manifest anxiety scale. Perceptual and motor skills, 65(1), 67-70.

Wolfe, C. D., \& Bell, M. A. (2014). Brain electrical activity of shy and non-shy preschool-aged children during executive function tasks. Infant and Child Development, 23(3), 259-272. https://doi.org/10.1002/icd.1858

Zhang, Y., Brady, M., \& Smith, S. (2001). Segmentation of Brain MR Images Through a Hidden Markov Random Field Model and the Expectation-Maximization Algorithm. IEEE Transactions on Medical Imaging, 20(1): 45-57. https://doi.org/10.1109/42.906424. 